\title{
Multigrid Methods for a Parameter Dependent Problem in Primal Variables
}

\author{
Joachim Schöberl* \\ joachim@numa.uni-linz.ac.at
}

Revision July, 1998

\begin{abstract}
In this paper we consider multigrid methods for the parameter dependent problem of nearly incompressible materials. We construct and analyze multilevel-projection algorithms, which can be applied to the mixed as well as to the equivalent, nonconforming finite element scheme in primal variables. For proper norms, we prove that the smoothing property and the approximation property hold with constants that are independent of the small parameter. Thus we obtain robust and optimal convergence rates for the $\mathrm{W}$-cycle and the variable $\mathrm{V}$-cycle multigrid methods. The numerical results pretty well conform the robustness and optimality of the multigrid methods proposed.
\end{abstract}

\section{Introduction}

We consider the linear elasticity problem to find $u \in\left[H_{0}^{1}(\Omega)\right]^{2}$ such that

$$
2 \mu \int_{\Omega} e(u): e(v) \mathrm{dx}+\lambda \int_{\Omega} \operatorname{div} u \operatorname{div} v \mathrm{dx}=\int_{\Omega} \tilde{f}^{T} v \mathrm{dx}
$$

with the positive constants $\lambda$ and $\mu$ of Lamé, the strain operator $e(u):=0.5\left(\nabla u+(\nabla u)^{T}\right)$ and the volume force $\tilde{f} \in\left[L_{2}(\Omega)\right]^{2}$. We are interested in the nearly incompressible case, i.e. the Poisson ration $\nu$ is close to 0.5 . Then the 'bad parameter' $\varepsilon:=2 \mu / \lambda$ becomes small.

For conforming low order finite element methods the parameter $\varepsilon$ enters disadvantageously into the discretization error estimate. This effect is also verified numerically and is well known as 'locking effect', [2]. Various non-conforming discretization methods lead to discretization errors robust for $\nu \rightarrow 0.5$, see [12], [10]. We use the mixed formulation for $u$ and $\operatorname{div} u$ to obtain a stable saddle-point system. By the choice of non-continuous finite elements for the dual variable, it can be eliminated at element level, and we return to a symmetric positive definite finite element method.

Also the convergence rate of standard multigrid methods applied to solve the positive definite linear system deteriorates as $\nu \rightarrow 0.5$. To overcome this difficulty, robust multigrid methods have been designed for the equivalent mixed finite element scheme with penalty

*This work is supported by the Austrian Science Fund - 'Fonds zur Förderung der wissenschaftlichen Forschung' - under project P 10643-TEC 
term in [21], [15], [3], [9], [4]. Related multigrid methods for the Stokes problem are analyzed in [8], [20]. The papers mainly differ in the kind of smoothing iteration used for the indefinite system.

In [18], a new multigrid method for parameter dependent problems in primal variables has been suggested and the analysis for the two-level method was given. The key components are an overlapping block-smoother capturing the divergence free basis functions, and a grid transfer operator prolongating coarse grid divergence free functions to fine grid divergence free functions. In this paper we establish the approximation and the smoothing property necessary for the multigrid analysis [14], [6].

During the analysis we switch between both equivalent algorithms, the primal one and the mixed one.

The outline of the paper is as follows. In Section 2 some available results are collected. The algorithmic aspects of the multigrid method are formulated in Section 3, the analysis is started in Section 4. Approximation property and smoothing property are proven in Section 5 and in Section 6, respectively. Numerical results are given in Section 7.

\section{Stability and Discretization}

We introduce the dual variable

$$
p:=\varepsilon^{-1} \operatorname{div} u
$$

and obtain the equivalent mixed problem to find $(u, p) \in X:=V \times Q:=\left[H_{0}^{1}(\Omega)\right]^{2} \times L_{2} / \mathbf{R}$ such that

$$
B((u, p),(v, q))=(f, v)_{0} \quad \forall(v, q) \in X,
$$

with $f=(2 \mu)^{-1} \tilde{f}$ and the bilinear-form

$$
B((u, p),(v, q))=(e(u), e(v))_{0}+(\operatorname{div} u, q)_{0}+(\operatorname{div} v, p)_{0}-\varepsilon(p, q)_{0},
$$

where $(., .)_{0}$ denotes the inner product in $L_{2}$ of scalar, vector valued or tensor valued functions. Clearly, $B$ is continuous on $X \times X$ with the product norm $\|(u, p)\|_{X}=\left(\|u\|_{1}^{2}+\|p\|_{0}^{2}\right)^{1 / 2}$. The proper stability criterion on some subspace $X_{*}:=V_{*} \times Q_{*} \subseteq X$ is the condition

$$
\sup _{(v, q) \in X_{*}} \frac{B((u, p),(v, q))}{\|(v, q)\|_{X}} \geq c\|(u, p)\|_{X} \quad \forall(u, p) \in X_{*} .
$$

Here and throughout the paper $\mathrm{c}$ will be a generic constant which is independent of the parameter $\varepsilon$ and the mesh-size defined below and which may be different in different equations. It follows from the second inequality of Korn, the LBB condition of the Stokes problem and further estimates due to the penalty term that $B$ is stable on $X_{*}=X$, see [11], [1]. We assume that $\Omega$ is a convex polygonal domain and get from [10] the regularity theorem

$$
\|u\|_{2}+\|p\|_{1} \leq c\|f\|_{0} .
$$

For finite element discretization we choose the subspace $X_{L}=V_{L} \times Q_{L} \subset X$, where $V_{L}$ consists of continuous, piecewise quadratic functions, and $Q_{L}$ of piecewise constant functions on a triangular mesh with mesh-size parameter $h_{L}$. The integer $L$ defines the number of multigrid levels. We get the finite dimensional problem find $\left(u_{L}, p_{L}\right) \in X_{L}$ such that

$$
B\left(\left(u_{L}, p_{L}\right),\left(v_{L}, q_{L}\right)\right)=\left(f, v_{L}\right)_{0} \quad \forall\left(v_{L}, q_{L}\right) \in X_{L} .
$$

The LBB condition is fulfilled for the pair of spaces $V_{L}$ and $Q_{L}$, see [11] p. 211, which implies the stability condition (4) on $X_{*}=X_{L}$. 
The essential fact is the non-continuity of the functions in $Q_{L}$ leading to an easily invertible matrix for the $L_{2}$ inner product. The dual variable $p_{L}$ can be eliminated elementwise and the problem can be reduced to the non-conforming symmetric and positive definite problem find $u_{L} \in V_{L}$ such that

$$
A_{L}\left(u_{L}, v_{L}\right)=\left(f, v_{L}\right)_{0} \quad \forall v_{L} \in V_{L},
$$

with the bilinear-form

$$
A_{L}(u, v)=(\varepsilon(u), \varepsilon(v))_{0}+\varepsilon^{-1}\left(I_{L}^{Q} \operatorname{div} u, \operatorname{div} v\right)_{0} .
$$

The operator $I_{L}^{Q}$ denotes the $L_{2}$-orthogonal projection onto $Q_{L}$. We mention that this projection can be implemented in the element matrix assembling subroutine. Due to the equivalence of the primal and the mixed finite element method we get bounds for the discretization error that are independent of $\varepsilon$ also for the primal version.

The author is aware of the sub-optimal convergence rate $O(h)$ for the $P_{2}-P_{0}$ element pairing. There exist several elements with non-continuous pressure and optimal convergence rate, see [13], [11]. The element is chosen for reasons of simpler notation and implementation, but the following analysis is not limited to the special element.

\section{The Multigrid Algorithm}

For the application of multigrid solvers a sequence of uniformly refined triangulations $\mathcal{T}_{l}$ of mesh-size $h_{l}$ and the corresponding nested $P_{2}-P_{0}$ finite element spaces

$$
X_{1}=V_{1} \times Q_{1} \subset X_{2}=V_{2} \times Q_{2} \subset \ldots \subset X_{L}=V_{L} \times Q_{L}
$$

are used. By means of the computable Q-orthogonal projection operators $I_{l}^{Q}: Q \rightarrow Q_{l}$ we define the bilinear-forms

$$
A_{l}(u, v)=(e(u), e(v))_{0}+\varepsilon^{-1}\left(I_{l}^{Q} \operatorname{div} u, \operatorname{div} v\right)_{0} \quad \forall u, v \in V,
$$

for $l=1, \ldots, L$. We mention that the forms are defined on the infinite dimensional space $V$. We define norms $\|u\|_{A_{l}}:=A_{l}(u, u)^{1 / 2}$ and the $L_{2}$ self-adjoint operators $A_{l}: V_{l} \rightarrow V_{l}$ as $\left(A_{l} u_{l}, v_{l}\right)_{0}=A_{l}\left(u_{l}, v_{l}\right), \forall u_{l}, v_{l} \in V_{l}, l=1, \ldots, L$. It is clear that $A_{l}(.,$.$) estimates A_{l+1}(.,$. from below, i.e.

$$
A_{l+1}(u, u) \geq A_{l}(u, u) \quad \forall u \in V,
$$

but the converse estimate does not hold with some constant independently bounded in $\varepsilon$.

This fact requires special grid transfer operators, which are constructed as follows. On each level $l=2, \ldots, L$ we define the subspace of functions which vanish on the boundaries of the coarse grid elements

$$
V_{l, T}:=\prod_{T \in \mathcal{T}_{l-1}}\left[H_{0}^{1}(T)\right]^{2} \cap V_{l} .
$$

It splits orthogonally into $\left|\mathcal{T}_{l-1}\right|$ subspaces. Each of them is generated by the basis functions belonging to the nodes inside a triangle of the coarser grid, see Figure 1.

We define the projection operator $P_{l, T}^{A_{l}}: V \rightarrow V_{l, T}$ such that

$$
A_{l}\left(P_{l, T}^{A_{l}} u, v_{l, T}\right)=A_{l}\left(u, v_{l, T}\right) \quad \forall u \in V, \forall v_{l, T} \in V_{l, T} .
$$




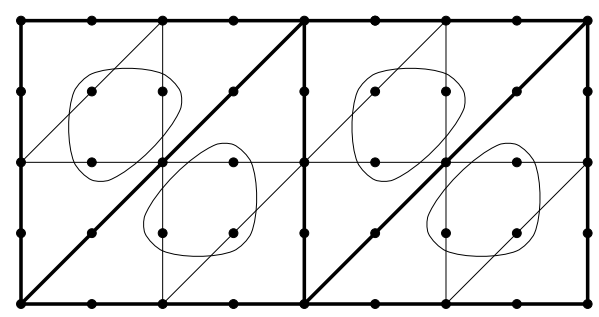

Figure 1: Subspace $V_{l, T}$ used in prolongation
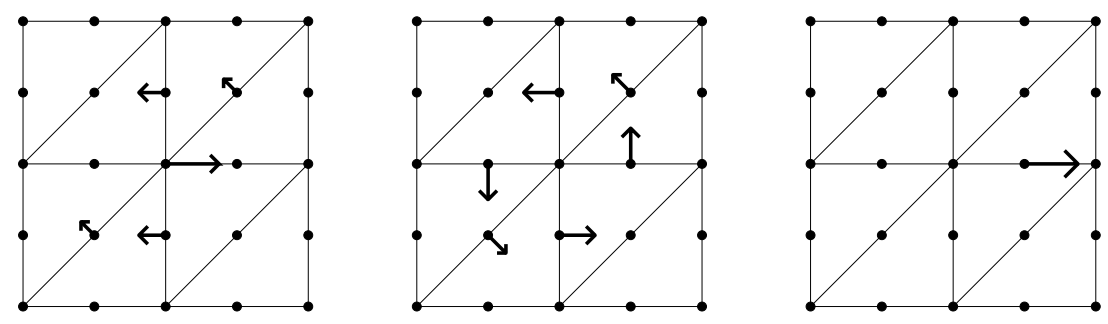

Figure 2: Basis functions for kernel of $I_{l}^{Q}$ div

The co-projection $I-P_{l T}^{A_{l}}$ is the discrete harmonic extension on each coarse grid triangle. It can be computed fast and will be used as prolongation operator. We use the natural embedding $V_{l-1} \subset V_{l}$ without denoting it by any symbol.

$$
\begin{gathered}
\text { Prolongation } u_{l-1} \rightarrow u_{l}: \\
u_{l}=\left(I-P_{l, T}^{A_{l}}\right) u_{l-1}
\end{gathered}
$$

The idea of this prolongation is to lift coarse grid div-free functions to fine grid div-free functions. As we will see later, this prolongation is continuous in the sense of

$$
\left\|\left(I-P_{l, T}^{A_{l}}\right) u_{l-1}\right\|_{A_{l}} \leq c\left\|u_{l-1}\right\|_{A_{l-1}} \quad \forall u_{l-1} \in V_{l-1} .
$$

We define the operator $E_{l}^{-1}: V_{l} \rightarrow V_{l}$ as $E_{l}^{-1}=P_{l, T}^{A_{l}} A_{l}^{-1}$ such that the prolongation can be rewritten as

$$
I-P_{l, T}^{A_{l}}=I-E_{l}^{-1} A_{l} .
$$

The operator $E_{l}$ is self-adjoint with respect to $(., .)_{0}$. In matrix form $E_{l}$ is the restriction of $A_{l}$ to the degrees of freedom spanning the space $V_{l, T}$. Using the $L_{2}$-orthogonal projection $P_{l-1}^{L_{2}}: V \rightarrow V_{l-1}$, the $(.,)_{0}$ - adjoint restriction operator is $P_{l-1}^{L_{2}}\left(I-A_{l} E_{l}^{-1}\right)$. We mention that the projection $P_{l-1}^{L_{2}}$ is required for notation only, it does not enter into the computation.

Also the smoother must be properly designed. A damped Richardson smoother $\left(I-\tau A_{l}\right)$ would need a damping parameter $\tau$ proportional to $\varepsilon$. Thus the components of the error in the kernel of $A_{l}$ would be smoothed out very slow, as $\varepsilon$ becomes small. The suggested smoother is a block Jacobi smoother, which takes care of the kernel of $I_{l}^{Q}$ div. On a simply connected domain with only one part of natural boundary conditions, the kernel of $I_{l}^{Q}$ div is spanned by basis functions drawn in Figure 2, similar to [11], pp 268.

These kernel basis functions are captured by subspaces $V_{l, i}$ generated by nodal basis functions belonging to the nodes drawn in Figure 3. For all $l=2, \ldots, L$, this leads to the definition of the $n_{l}$ subspaces

$$
V_{l, i}=\left[H_{0}^{1}\left(\Omega_{i}\right)\right]^{2} \cap V_{l}
$$




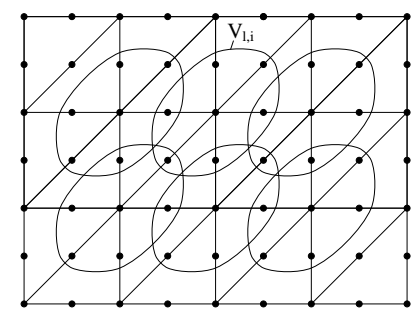

Figure 3: Subspaces containing div-free basis functions

assigned to the corner nodes $N_{l, i}, i=1, \ldots, n_{l}$, of the triangulation $\mathcal{T}_{l}$. Here, $\Omega_{i}$ is the closure of the union over elements in $\mathcal{T}_{l}$ adjacent to the node $N_{l, i}$. We define the projections $P_{l, i}^{A_{l}}: V \rightarrow V_{l, i}$ as

$$
A_{l}\left(P_{l, i}^{A_{l}} u, v_{l, i}\right)=A_{l}\left(u, v_{l, i}\right) \quad \forall u \in V \quad \forall v_{l, i} \in V_{l, i}
$$

By means of these subspaces we define the block Jacobi smoother as

$$
\begin{array}{r}
\text { Smoother } S_{l}^{A}: V_{l} \rightarrow V_{l}: \\
S_{l}^{A}=I-\tau \sum_{i=1}^{n_{l}} P_{l, i}^{A_{l}}
\end{array}
$$

The necessary damping parameter depends only on the number of overlapping spaces, which is bounded for shape regular elements. Especially, it does not depend on $\varepsilon$ and $l$. We assume that $\tau$ is small enough to ensure only positive eigenvalues of $S_{l}^{A}$. In the numerical examples, we will also use the according block Gauss-Seidel smoother, which does not need any damping at all. We also define the operator $D_{l}^{-1}: V_{l} \rightarrow V_{l}$ as

$$
D_{l}^{-1}=\sum_{i=1}^{n_{l}} P_{l, i}^{A_{l}} A_{l}^{-1}
$$

which corresponds in matrix form to the sum over local inverses. By means of $D_{l}$ the smoother can be written as

$$
S_{l}^{A}=I-\tau D_{l}^{-1} A_{l} .
$$

Now we can state the multigrid algorithm in recursive form. We apply $m_{l}$ smoothing steps on the level $l$ and perform either the $V$-cycle $(q=1)$ or the $W$-cycle $(q=2)$.

\section{Algorithm 1}

Procedure $M G(u, f, l)$

if $l=1$

$$
M G(u, f, l)=A_{1}^{-1} f
$$

else

$$
\begin{aligned}
& u^{1,0}=u \\
& \text { do } j=1, \ldots, m_{l} \\
& \quad u^{1, j}=u^{1, j-1}+\tau D_{l}^{-1}\left(f-A_{l} u^{1, j-1}\right) \\
& d^{1}=f-A_{l} u^{1, m_{l}} \\
& d^{2}=P_{l-1}^{L_{2}}\left(I-A_{l} E_{l}^{-1}\right) d^{1} \\
& u^{2,0}=0
\end{aligned}
$$




$$
\begin{aligned}
& \operatorname{dos}=1, \ldots, q \\
& \quad u^{2, j}=M G\left(u^{2, j-1}, d^{2}, l-1\right) \\
& u^{3}=\left(I-E_{l}^{-1} A_{l}\right) u^{2, q} \\
& u^{4,0}=u^{1, m_{l}}+u^{3} \\
& \operatorname{do} j=1, \ldots, m_{l} \\
& \quad u^{4, j}=u^{4, j-1}+\tau D_{l}^{-1}\left(f-A_{l} u^{4, j-1}\right) \\
& M G(u, f, l)=u^{4, m_{l}}
\end{aligned}
$$

\section{The Multigrid Analysis}

In this section we start the analysis of the multigrid method. First, we specify a multigrid method for the mixed form and prove equivalence to the algorithm stated in the last chapter. We define an $L_{2}$-like norm, for which we will prove the approximation property and the smoothing property in the following two chapters.

For $l=1, \ldots, L$ we define the subspaces

$$
X_{l, 0}:=\left\{\left(u_{l}, p_{l}\right) \in X_{l}: I_{l}^{Q} \operatorname{div} u_{l}=\varepsilon p_{l}\right\} .
$$

We will use the relation

$$
B\left(\left(u_{l}, p_{l}\right),\left(0, q_{l}\right)\right)=0 \quad \forall\left(u_{l}, p_{l}\right) \in X_{l, 0}, \quad \forall q_{l} \in Q_{l}
$$

later. From (10) and (19) it follows that

$$
B\left(\left(u_{l}, p_{l}\right),\left(v_{l}, 0\right)\right)=A_{l}\left(u_{l}, v_{l}\right) \quad \forall\left(u_{l}, p_{l}\right) \in X_{l, 0}, \quad \forall v_{l} \in V_{l}
$$

is valid. We extend definition (14) to local mixed spaces

$$
X_{l, i}:=V_{l, i} \times Q_{l, i} \quad \text { with } \quad Q_{l, i}=\left(L_{2}\left(\Omega_{l, i}\right) / \mathbf{R}\right) \cap Q_{l}
$$

for $i=1, \ldots, n_{l}, l=1, \ldots, L$. We also extend the spaces $V_{l, T}$ of the prolongation defined in (11) to the mixed spaces

$$
X_{l, T}:=V_{l, T} \times Q_{l, T} \quad \text { with } \quad Q_{l, T}:=\prod_{T \in \mathcal{T}_{l-1}}\left(L_{2}(T) / \mathbf{R}\right) \cap Q_{l},
$$

$l=2, \ldots, L$. The spaces are designed such that the orthogonal decomposition

$$
Q_{l}=Q_{l-1} \oplus Q_{l, T}
$$

and

$$
B\left(\left(u_{T}, p_{T}\right),\left(0, q_{l-1}\right)\right)=0 \quad \forall\left(u_{T}, p_{T}\right) \in X_{l, T}, \quad \forall q_{l-1} \in Q_{l-1}
$$

hold. In addition to the norm $\|.\|_{X}$ we define the energy-norm

$$
\|(u, p)\|_{B}:=B((u, p),(u,-p))^{1 / 2}=\left(\|e(v)\|_{0}^{2}+\varepsilon\|p\|_{0}^{2}\right)^{1 / 2},
$$

which by Korn's inequality is equivalent to the norm $\left(\|u\|_{1}^{2}+\varepsilon\|p\|_{0}^{2}\right)^{1 / 2}$.

For any subspace $X_{*} \subset X$ the projection $P_{*}^{B}: X \rightarrow X_{*}$ is defined by

$$
B\left(P_{*}^{B}(u, p),(v, q)\right)=B((u, p),(v, q)) \quad \forall(v, q) \in X_{*} .
$$

We will use projections to $X_{l-1}, X_{l, T}$ and $X_{l, i}$. The next lemma collects some of their properties. 


\section{Lemma 1}

1. All subspaces $X_{l}, X_{l, i}$ and $X_{l, T}$ fulfill the stability condition (4) with one common constant $c$.

2. The projections $P_{l-1}^{B}, P_{l, i}^{B}, P_{l, T}^{B}$ are well defined and uniformly bounded on $X_{l}$ with respect to the $\|\cdot\|_{X}$-norm.

3. $P_{l-1}^{B} \operatorname{maps} X_{l, 0}$ into $X_{l-1,0}$.

4. $P_{l, i}^{B}$ and $P_{l, T}^{B}$ map $X_{l, 0}$ into itself, and they are bounded by 1 with respect to the $\|.\|_{B}$-norm on $X_{l, 0}$.

5. The co-projection $I-P_{l, T}^{B}$ maps $X_{l-1,0}$ into $X_{l, 0}$.

Proof: 1 . Stability condition for the spaces are standard. The spaces $V_{l, i}$ and the factorspaces of $V_{l, T}$ can be derived from a finite number of spaces by scaling and translation, and these transformations do not change the stability constant. Thus the common constant is the maximum of a finite number of stability constants.

2. The continuity of $P_{*}^{B}$ with respect to $\|.\|_{X^{-}}$norm follows from the stability condition (4)

$$
\left\|P_{*}^{B}(u, p)\right\|_{X} \leq c \sup _{(v, q) \in X_{*}} \frac{B\left(P_{*}^{B}(u, p),(v, q)\right)}{\|(v, q)\|_{X}}=c \sup _{(v, q) \in X_{*}} \frac{B((u, p),(v, q))}{\|(v, q)\|_{X}} \leq c\|(u, p)\|_{X} .
$$

3. For $(u, p) \in X_{l, 0}$ we get

$$
B\left(P_{l-1}^{B}(u, p),\left(0, q_{l-1}\right)\right)=B\left((u, p),\left(0, q_{l-1}\right)\right)=0 \quad \forall q_{l-1} \in Q_{l-1} .
$$

4. Next set $(\hat{u}, \hat{p})=P_{l, i}^{B}(u, p)$. We now decompose a function $q \in Q_{l}$ orthogonally into $q_{1} \in Q_{l, i}$ and $q_{2}=(q, 1)_{0, \Omega_{l, i}} /\left|\Omega_{l, i}\right|$ in $\Omega_{l, i}$ and $q_{2}=q$ in $\Omega \backslash \Omega_{l, i}$. $B\left((\hat{u}, \hat{p}),\left(0, q_{1}\right)\right)$ vanishes by definition of the projection, $B\left((\hat{u}, \hat{p}),\left(0, q_{2}\right)\right)=\left(\operatorname{div} \hat{u}, q_{2}\right)_{0, \Omega_{l, i}}-\varepsilon\left(\hat{p}, q_{2}\right)_{0, \Omega_{l, i}}=0$ is achieved by Green's theorem and definition of $Q_{l, i}$. By (24), (25) and the same arguments $P_{l, T}^{B} \in X_{l, 0}$ is proven.

Now, let $(u, p) \in X_{l, 0}$ and $X_{*}$ such that $(\hat{u}, \hat{p})=P_{*}^{B}(u, p) \in X_{l, 0}$. Then,

$$
\begin{aligned}
& \|(\hat{u}, \hat{p})\|_{B}^{2}=B((\hat{u}, \hat{p}),(\hat{u},-\hat{p}))=B((u, p),(\hat{u},-\hat{p}))= \\
& \quad=B((u, p),(\hat{u},-\hat{p}))+B((u, p),(0, \hat{p}))-B((0, p),(\hat{u}, \hat{p}))=(e(u), e(\hat{u}))_{0}+\varepsilon(p, \hat{p})_{0} \\
& \quad \leq\left(\|e(u)\|_{0}^{2}+\varepsilon\|p\|_{0}^{2}\right)^{1 / 2}\left(\|e(\hat{u})\|_{0}^{2}+\varepsilon\|\hat{p}\|_{0}^{2}\right)^{1 / 2}=\|(u, p)\|_{B}\|(\hat{u}, \hat{p})\|_{B}
\end{aligned}
$$

gives the upper bound 1 .

5. Let $(u, p) \in X_{l-1,0}$. Then

$$
B\left(\left(I-P_{l, T}^{B}\right)(u, p),\left(0, q_{l-1}\right)\right)=0 \quad \forall q_{l-1} \in Q_{l-1}
$$

holds because of the assumption and (25). The same form tested with $q \in Q_{l, T}$ vanishes because of the definition of the projection, and $Q_{l}$ can be decomposed by (24).

We also define projections $P_{*}^{A_{l}, A_{k}}: V_{k} \rightarrow V_{*} \subset V_{l}$ by

$$
A_{l}\left(P_{*}^{A_{l}, A_{k}} u, v\right)=A_{k}(u, v) \quad \forall v \in V_{*}
$$

and set $P_{*}^{A_{l}}=P_{*}^{A_{l}, A_{l}}$. This definition is consistent with (12) and (15).

\section{Lemma 2}

Let $X_{*}=V_{*} \times Q_{*} \subset X_{l}$ and $(\hat{u}, \hat{p})=P_{*}^{B}(u, p)$. 
1. If $(u, p) \in X_{k, 0}$ and $(\hat{u}, \hat{p}) \in X_{l, 0}$, then $\hat{u}=P_{*}^{A_{l}, A_{k}} u$.

2. If $(u, p)-(\hat{u}, \hat{p}) \in X_{l, 0}$, then $\hat{u}=P_{*}^{A_{l}} u$.

Proof: To verify statement 1 we use (21) and obtain

$$
A_{l}(\hat{u}, v)=B((\hat{u}, \hat{p}),(v, 0))=B((u, p),(v, 0))=A_{k}(u, v) \quad \forall v \in V_{*} .
$$

Statement 2 is checked by

$$
A_{l}(\hat{u}-u, v)=B((\hat{u}-u, \hat{p}-p),(v, 0))=0 \quad \forall v \in V_{*} .
$$

Algorithm 1 leads to the multigrid operator $M_{L}^{A}$, which for $l=2, \ldots, L$ fulfills the recursion

$$
\begin{aligned}
& M_{1}^{A}=0 \\
& M_{l}^{A}=\left(S_{l}^{A}\right)^{m_{l}}\left(I-\left(I-P_{l, T}^{A_{l}}\right)\left(I-\left(M_{l-1}^{A}\right)^{q}\right) P_{l-1}^{A_{l-1}, A_{l}}\left(I-P_{l, T}^{A_{l}}\right)\right)\left(S_{l}^{A}\right)^{m_{l}} .
\end{aligned}
$$

We define the corresponding multigrid operator for the mixed system as

$$
\begin{aligned}
& M_{1}^{B}=0, \\
& M_{l}^{B}=\left(S_{l}^{B}\right)^{m_{l}}\left(I-\left(I-P_{l, T}^{B}\right)\left(I-\left(M_{l-1}^{B}\right)^{q}\right) P_{l-1}^{B}\left(I-P_{l, T}^{B}\right)\right)\left(S_{l}^{B}\right)^{m_{l}},
\end{aligned}
$$

with the smoothing operators

$$
S_{l}^{B}:=I-\tau \sum_{i=1}^{n_{l}} P_{l, i}^{B}
$$

for $2 \leq l \leq L$. The iteration for the mixed system is well defined for the limit case $\varepsilon=0$, too.

\section{Theorem 1 (Equivalence of Algorithms)}

Both multigrid procedures are equivalent, namely for $\left(u_{l}, p_{l}\right) \in X_{l, 0}$ there holds

$$
\left(\hat{u}_{l}, \hat{p}_{l}\right):=M_{l}^{B}\left(u_{l}, p_{l}\right) \quad \text { fulfills } \quad \hat{u}_{l}=M_{l}^{A}\left(u_{l}\right) .
$$

Proof: By induction on $l$, Lemma 1 and Lemma 2.

In the following chapters we will need the approximation properties of the finite element spaces. Let $I_{l}^{V}$ be the Lagrange interpolator into $V_{l}$. Recall the (local) $L_{2}$ projector $I_{l}^{Q}$ to $Q_{l}$ and define the product operator $I_{l}^{X}=\left(I_{l}^{V}, I_{l}^{Q}\right): X \rightarrow X_{l}$. Then the approximation inequalities

$$
\left\|u-I_{l}^{V} u\right\|_{1} \leq c h_{l}\|u\|_{2} \quad \text { and } \quad\left\|p-I_{l}^{Q} p\right\|_{0} \leq c h_{l}\|p\|_{1}
$$

hold.

We define the norm

$$
\|(u, p)\|_{l, 0}^{2}:=h_{l}^{-2}\|u\|_{0}^{2}+\varepsilon\|p\|_{0}^{2}+\left\|I_{l-1}^{Q} p\right\|_{0}^{2} .
$$

On the space $X_{l, 0}$, it is identical to the norm

$$
\|u\|_{l, 0}^{2}:=h_{l}^{-2}\|u\|_{0}^{2}+\varepsilon^{-1}\left\|I_{l}^{Q} \operatorname{div} u\right\|_{0}^{2}+\varepsilon^{-2}\left\|I_{l-1}^{Q} \operatorname{div} u\right\|_{0}^{2}
$$

on $V_{l}$. These norms will be used in the multigrid proof for measuring smoothness.

The main theorem of this paper is 


\section{Theorem 2 (Two-Grid Convergence)}

The two-grid operator $\hat{M}_{l}^{A}$ can be estimated by

$$
\left\|\hat{M}_{l}^{A}\right\|_{A_{l}} \leq c m_{l}^{-1 / 4}
$$

with a constant $c$ independent of $l$ and $\varepsilon$. The two-grid operator $\hat{M}_{l}^{B}$ maps $X_{l, 0}$ into itself and is bounded on $X_{l, 0}$ by

$$
\left\|\hat{M}_{l}^{B}\right\|_{B} \leq c m_{l}^{-1 / 4}
$$

with a constant $c$ independent of $l$ and $\varepsilon$.

Proof: Define for $\left(u^{0}, p^{0}\right) \in X_{l, 0}$

$$
\left(u^{1}, p^{1}\right)=\left(I-\left(I-P_{l, T}^{B}\right) P_{l-1}^{B}\left(I-P_{l, T}^{B}\right)\right)\left(u^{0}, p^{0}\right)
$$

and

$$
\left(u^{2}, p^{2}\right)=\left(S_{l}^{B}\right)^{m_{l}}\left(u^{1}, p^{1}\right) .
$$

By the previous lemmata we get $\left(u^{1}, p^{1}\right) \in X_{l, 0}$ and

$$
u^{1}=\left(I-\left(I-P_{l, T}^{A_{l}}\right) P_{l-1}^{A_{l-1}, A_{l}}\left(I-P_{l, T}^{A_{l}}\right)\right) u^{0} .
$$

In Section 5 we will prove the approximation property (see Theorem 4)

$$
\left\|\left(u^{1}, p^{1}\right)\right\|_{l, 0}=\left\|u^{1}\right\|_{l, 0} \leq c\left\|u^{0}\right\|_{A_{l}}=c\left\|\left(u^{0}, p^{0}\right)\right\|_{B}
$$

using the mixed form. We also get $\left(u^{2}, p^{2}\right) \in X_{l, 0}$ and

$$
u^{2}=\left(S_{l}^{A}\right)^{m_{l}} u^{1} .
$$

In Section 6 we will prove the smoothing property (see Theorem 5)

$$
\left\|\left(u^{2}, p^{2}\right)\right\|_{B}=\left\|u^{2}\right\|_{A_{l}} \leq c m^{-1 / 4}\left\|u^{1}\right\|_{l, 0}=c m^{-1 / 4}\left\|\left(u^{1}, p^{1}\right)\right\|_{l, 0}
$$

using the primal form. Combining both properties proves the theorem.

The following theorem follows by standard techniques [14], [6], [5].

\section{Theorem 3 (Multigrid Convergence)}

- The norm of the $W$-cycle operator is bounded independently of $L$ and $\varepsilon$ if the number of smoothing steps $m_{l}$ is sufficiently large.

- The variable $V$-cycle operator with $m^{l}=2^{L-l}$ leads to a preconditioner $C_{L}^{-1}:=(I-$ $\left.M_{L}^{A}\right) A_{L}^{-1}$ with condition number $\kappa\left(C_{L}^{-1} A_{L}\right)$ bounded independently of $L$ and $\varepsilon$.

\section{Approximation Property}

The coarse grid operator $\left(u_{1}, p_{1}\right) \in X_{l, 0} \rightarrow\left(u_{5}, p_{5}\right) \in X_{l, 0}$ is split into

$$
\begin{aligned}
& \left(u_{2}, p_{2}\right)=\left(I-P_{l, T}^{B}\right)\left(u_{1}, p_{1}\right), \\
& \left(u_{3}, p_{3}\right)=P_{l-1}^{B}\left(u_{2}, p_{2}\right), \\
& \left(u_{4}, p_{4}\right)=\left(I-P_{l, T}^{B}\right)\left(u_{3}, p_{3}\right), \\
& \left(u_{5}, p_{5}\right)=\left(u_{1}, p_{1}\right)-\left(u_{4}, p_{4}\right) .
\end{aligned}
$$


Theorem 4 (Approximation Property)

Let $\left(u_{1}, p_{1}\right) \in X_{l, 0}$ and compute $\left(u_{5}, p_{5}\right)$ by (39). Then the approximation property

$$
\left\|\left(u_{5}, p_{5}\right)\right\|_{l, 0} \leq c\left\|\left(u_{1}, p_{1}\right)\right\|_{B}
$$

is valid.

Proof: We use the triangle inequality and the three lemmata proven below to obtain the result

$$
\begin{aligned}
& \left\|\left(u_{5}, p_{5}\right)\right\|_{l, 0}=\left\|\left(u_{1}, p_{1}\right)-\left(u_{4}, p_{4}\right)\right\|_{0} \\
& \quad \leq\left\|\left(u_{1}, p_{1}\right)-\left(u_{2}, p_{2}\right)\right\|_{l, 0}+\left\|\left(u_{2}, p_{2}\right)-\left(u_{3}, p_{3}\right)\right\|_{l, 0}+\left\|\left(u_{3}, p_{3}\right)-\left(u_{4}, p_{4}\right)\right\|_{l, 0} \\
& \quad \leq c\left\|\left(u_{1}, p_{1}\right)\right\|_{B} .
\end{aligned}
$$

\section{Lemma 3}

With the notation of (39) there holds

$$
\left\|\left(u_{2}, p_{2}\right)\right\|_{B}+\left\|\left(u_{2}, p_{2}\right)-\left(u_{1}, p_{1}\right)\right\|_{l, 0}+\left\|p_{2}-I_{l-1}^{Q} p_{2}\right\|_{0} \leq c\left\|\left(u_{1}, p_{1}\right)\right\|_{B} .
$$

Proof: Lemma 1 gives $\left\|P_{l, T}^{B}\left(u_{1}, p_{1}\right)\right\|_{B} \leq\left\|\left(u_{1}, p_{1}\right)\right\|_{B}$, which bounds the first term. The second term is bounded due to the norm equivalence $\|.\|_{B} \sim\|\cdot\|_{l, 0}$ on $X_{l, T}$. From $p_{2}-I_{l-1}^{Q} p_{2} \in$ $Q_{l, T}$, stability (4) of $X_{l, T}$, orthogonality (25) and the definition of $P_{l, T}^{B}$ we obtain

$$
\begin{aligned}
& \left\|p_{2}-I_{l-1}^{Q} p_{2}\right\|_{0} \leq c \sup _{(v, q) \in Q_{l, T}} \frac{B\left(\left(0, p_{2}-I_{l-1}^{Q} p_{2}\right),(v, q)\right)}{\|(v, q)\|_{X}} \\
& =c \sup _{(v, q) \in Q_{l, T}} \frac{B\left(\left(0, p_{2}\right),(v, q)\right)}{\|(v, q)\|_{X}}=c \sup _{(v, q) \in Q_{l, T}} \frac{B\left(\left(-u_{2}, 0\right),(v, q)\right)}{\|(v, q)\|_{X}} \leq c\left\|u_{2}\right\|_{1} .
\end{aligned}
$$

\section{Lemma 4}

With the notation of (39) there holds

$$
\left\|\left(u_{3}, p_{3}\right)\right\|_{B}+\left\|\left(u_{3}, p_{3}\right)-\left(u_{2}, p_{2}\right)\right\|_{l, 0} \leq c\left\|\left(u_{1}, p_{1}\right)\right\|_{B} .
$$

Proof: By stability of $X_{l-1}$, the definition of $\left(u_{3}, p_{3}\right)$, continuity of $B(.,$.$) and Lemma 3$ we get

$$
\begin{aligned}
\left\|u_{3}\right\|_{1}+\left\|p_{3}-I_{l-1}^{Q} p_{2}\right\|_{0} & \leq c \sup _{(v, q) \in Q_{l-1}} \frac{B\left(\left(u_{3}, p_{3}-I_{l-1}^{Q} p_{2}\right),(v, q)\right)}{\|(v, q)\|_{X}} \\
& =c \sup _{(v, q) \in Q_{l-1}} \frac{B\left(\left(u_{2}, p_{2}-I_{l-1}^{Q} p_{2}\right),(v, q)\right)}{\|(v, q)\|_{X}} \\
& \leq c\left(\left\|u_{2}\right\|_{1}+\left\|p_{2}-I_{l-1}^{Q} p_{2}\right\|_{0}\right) \leq c\left\|\left(u_{1}, p_{1}\right)\right\|_{B},
\end{aligned}
$$

and in combination with Lemma 3

$$
\left\|p_{3}-p_{2}\right\|_{0} \leq\left\|p_{3}-I_{l-1}^{Q} p_{2}\right\|_{0}+\left\|p_{2}-I_{l-1}^{Q} p_{2}\right\|_{0} \leq c\left\|\left(u_{1}, p_{1}\right)\right\|_{B} .
$$

This gives also

$$
\varepsilon\left\|p_{3}\right\|_{0}^{2} \leq 2 \varepsilon\left\|p_{2}-p_{3}\right\|_{0}^{2}+2 \varepsilon\left\|p_{2}\right\|_{0}^{2} \leq c\left\|\left(u_{1}, p_{1}\right)\right\|_{B}^{2} .
$$


We state the dual problem on $X$

$$
B((\varphi, \psi),(v, q))=\left(u_{2}-u_{3}, v\right)_{0} \quad \forall(v, q) \in X
$$

and get the $L_{2}$ estimate by Galerkin orthogonality, approximation (30) and regularity (5)

$$
\begin{aligned}
\left\|u_{2}-u_{3}\right\|_{0}^{2} & =B\left((\varphi, \psi),\left(u_{2}-u_{3}, p_{2}-p_{3}\right)\right) \\
& =B\left((\varphi, \psi)-\left(I_{l-1}^{V} \varphi, I_{l-1}^{Q} \psi\right),\left(u_{2}-u_{3}, p_{2}-p_{3}\right)\right) \\
& \leq c\left(\left\|\varphi-I_{l-1}^{V} \varphi\right\|_{1}+\left\|\psi-I_{l-1}^{Q} \psi\right\|_{0}\right)\left(\left\|u_{2}-u_{3}\right\|_{1}+\left\|p_{2}-p_{3}\right\|_{0}\right) \\
& \leq c h\left(\|\varphi\|_{2}+\|\psi\|_{1}\right)\left(\left\|u_{2}\right\|_{1}+\left\|u_{3}\right\|_{1}+\left\|p_{2}-p_{3}\right\|_{0}\right) \\
& \leq c h\left\|u_{2}-u_{3}\right\|_{0}\left\|\left(u_{1}, p_{1}\right)\right\|_{B} .
\end{aligned}
$$

Dividing by $\left\|u_{2}-u_{3}\right\|_{0}$ we obtain the result.

\section{Lemma 5}

With the notation of (39) there holds

$$
\left\|\left(u_{4}, p_{4}\right)-\left(u_{3}, p_{3}\right)\right\|_{l, 0} \leq c\left\|\left(u_{1}, p_{1}\right)\right\|_{B} .
$$

Proof: Friedrichs' inequality on $V_{l, T}$, stability (4), Galerkin - and orthogonality (25) give

$$
\begin{aligned}
& \left\|\left(u_{4}, p_{4}\right)-\left(u_{3}, p_{3}\right)\right\|_{l, 0} \leq c\left\|\left(u_{4}, p_{4}\right)-\left(u_{3}, p_{3}\right)\right\|_{X} \\
& \quad \leq c \sup _{(v, q) \in X_{l, T}} \frac{B\left(\left(u_{3}-u_{4}, p_{3}-p_{4}\right),(v, q)\right)}{\|(v, q)\|_{X}}=c \sup _{(v, q) \in X_{l, T}} \frac{B\left(\left(u_{3}, p_{3}\right),(v, q)\right)}{\|(v, q)\|_{X}} \\
& \quad=c \sup _{(v, q) \in X_{l, T}} \frac{B\left(\left(u_{3}, 0\right),(v, q)\right)}{\|(v, q)\|_{X}} \leq\left\|u_{3}\right\|_{V},
\end{aligned}
$$

and the proof if complete.

\section{Smoothing Property}

In this chapter we prove the smoothing property

$$
\left\|\left(I-\tau D_{l}^{-1} A_{l}\right)^{m} u\right\|_{A_{l}} \leq c m^{-1 / 4}\|u\|_{l, 0}
$$

Recall that we have chosen $\tau$ such that $\left\|\tau D_{l}^{-1} A_{l}\right\|_{A_{l}} \leq 1$. The estimate

$$
\left\|\left(I-\tau D_{l}^{-1} A_{l}\right)^{m} u\right\|_{A_{l}}^{2}=\left(D_{l}^{-1} A_{l}\left(I-\tau D_{l}^{-1} A_{l}\right)^{2 m} u, u\right)_{D_{l}} \leq c m^{-1}\|u\|_{D_{l}}^{2}
$$

is well established in multigrid theory [14].

By additive Schwarz techniques [22], [17] the induced norm $\|u\|_{D_{l}}=\left(D_{l} u, u\right)_{0}^{1 / 2}$ can be expressed by

$$
\|u\|_{D_{l}}^{2}=\inf _{\substack{u=\sum_{u, i} u_{l, i} \\ u_{l, i} \in V_{l, i}}} \sum\left\|u_{l, i}\right\|_{A_{l}}^{2} .
$$

If the estimate $\|u\|_{D_{l}} \leq c\|u\|_{l, 0}$ would be true, the smoothing property would be proven. Unfortunately, it is not. The essential part of this section is the proof of the estimate

$$
\|u\|_{\left[D_{l}, A_{l}\right]_{1 / 2}} \leq c\|u\|_{l, 0}
$$


where $\|\cdot\|_{\left[D_{l}, A_{l}\right]_{1 / 2}}$ is the interpolation norm between $\|\cdot\|_{D_{l}}$ and $\|\cdot\|_{A_{l}}$ with parameter $1 / 2$. We use the real method of interpolation of Lions and Peetre [16], see also [7]. Inequalities (45) and (46) immediately give the smoothing property

$$
\begin{aligned}
& \left\|\left(I-\tau D_{l}^{-1} A_{l}\right)^{m} u\right\|_{A_{l}} \leq c\left\|\left(I-\tau D_{l}^{-1} A_{l}\right)^{m} u\right\|_{\left[A_{l}, A_{l}\right]_{1 / 2}} \\
& \quad \leq c m^{-1 / 4}\|u\|_{\left[D_{l}, A_{l}\right]_{1 / 2}} \leq c m^{-1 / 4}\|u\|_{l, 0} .
\end{aligned}
$$

We define the bilinear-form for the limit case $\varepsilon=0$ as

$$
B_{0}((u, p),(v, q))=(e(u), e(v))_{0}+(\operatorname{div} u, q)_{0}+(\operatorname{div} v, p)_{0} .
$$

To establish (46) we split $u=u_{1}+u_{2}+u_{3}$ by solving for $\left(u_{i}, p_{i}\right) \in X_{l}$ such that

$$
\begin{aligned}
& B_{0}\left(\left(u_{1}, p_{1}\right),(v, q)\right)=B_{0}((u, 0),(v, 0)) \\
& B_{0}\left(\left(u_{2}, p_{2}\right),(v, q)\right)=B_{0}\left((u, 0),\left(0, q-I_{l-1}^{Q} q\right)\right), \\
& B_{0}\left(\left(u_{3}, p_{3}\right),(v, q)\right)=B_{0}\left((u, 0),\left(0, I_{l-1}^{Q} q\right)\right) \quad \forall(v, q) \in X_{l} .
\end{aligned}
$$

The splitting is constructed such that $u_{1}$ is discrete divergence free, $u_{2}$ has non-smooth divergence and $u_{3}$ has smooth divergence.

\section{Theorem 5 (Smoothing Property)}

The estimate (46) and therefore the smoothing property (44) are valid.

Proof: We split $u$ using (49), apply the triangle inequality, Lemma 7 - 9, and Lemma 6 below to obtain (46) by

$$
\begin{aligned}
\|u\|_{\left[D_{l}, A_{l}\right]_{1 / 2}} & \leq\left\|u_{1}\right\|_{\left[D_{l}, A_{l}\right]_{1 / 2}}+\left\|u_{2}\right\|_{\left[D_{l}, A_{l}\right]_{1 / 2}}+\left\|u_{3}\right\|_{\left[D_{l}, A_{l}\right]_{1 / 2}} \\
& \leq c\left(\left\|u_{1}\right\|_{l, 0}+\left\|u_{2}\right\|_{l, 0}+\left\|u_{3}\right\|_{l, 0}\right) \\
& \leq c\|u\|_{l, 0} .
\end{aligned}
$$

The smoothing property (44) follows by the estimates (47).

\section{Lemma 6}

The decomposition (49) is stable in $\|.\|_{l, 0}$ norm, namely

$$
\left\|u_{1}\right\|_{l, 0}+\left\|u_{2}\right\|_{l, 0}+\left\|u_{3}\right\|_{l, 0} \leq c\|u\|_{l, 0} .
$$

Proof: By stability (4) we get the bounds $\left\|u_{1}\right\|_{1}+\left\|p_{1}\right\|_{0} \leq c\|u\|_{1}$ and $\left\|u_{2}\right\|_{1}+\left\|p_{2}\right\|_{0} \leq$ $c\left\|I_{l}^{Q} \operatorname{div} u\right\|_{0}$. First, we bound $\left\|u_{1}\right\|_{l, 0}^{2}=h^{-2}\left\|u_{1}\right\|_{0}^{2}$. The solution of the dual problem find $(\varphi, \psi) \in X$ such that

$$
B_{0}((\varphi, \psi),(v, q))=\left(u_{1}, v\right)_{0} \quad \forall(v, q) \in X,
$$

is bounded by $\|\varphi\|_{2}+\|\psi\|_{1} \leq c\left\|u_{1}\right\|_{0}$. By Galerkin orthogonality, approximation, regularity, and the inverse inequality $h\|u\|_{1} \leq c\|u\|_{0}$ we obtain

$$
\begin{aligned}
& \left\|u_{1}\right\|_{0}^{2}=B_{0}\left((\varphi, \psi),\left(u_{1}, p_{1}\right)\right) \\
& \quad=B_{0}\left((\varphi, \psi)-I_{l}^{X}(\varphi, \psi),\left(u_{1}, p_{1}\right)\right)+B_{0}\left(I_{l}^{X}(\varphi, \psi)-(\varphi, \psi),(u, 0)\right)+B_{0}((\varphi, \psi),(u, 0)) \\
& \leq c\left(h\left(\|\varphi\|_{2}+\|\psi\|_{1}\right)\left(\left\|u_{1}\right\|_{1}+\left\|p_{1}\right\|_{0}\right)+h\left(\|\varphi\|_{2}+\|\psi\|_{1}\right)\|u\|_{1}+\left(\|\varphi\|_{2}+\|\psi\|_{1}\right)\|u\|_{0}\right) \\
& \quad \leq c\left(h\left\|u_{1}\right\|_{0}\|u\|_{1}+\left\|u_{1}\right\|_{0}\|u\|_{0}\right) \leq c\left\|u_{1}\right\|_{0}\|u\|_{0} .
\end{aligned}
$$


Next, we estimate

$$
h^{-2}\left\|u_{2}\right\|_{0}^{2} \leq c\left\|I_{l}^{Q} \operatorname{div} u\right\|_{0}^{2} \leq c \varepsilon\|u\|_{l, 0}^{2}
$$

Therefore let

$$
B_{0}((\varphi, \psi),(v, q))=\left(u_{2}, v\right)_{0} \quad \forall(v, q) \in X,
$$

then we get by $B\left(\left(u_{2}, p_{2}\right),\left(v_{l}, q_{l-1}\right)\right)=0 \quad \forall v_{l} \in V_{l}, q_{l-1} \in Q_{l-1}$

$$
\begin{aligned}
& \left\|u_{2}\right\|_{0}^{2}=B_{0}\left((\varphi, \psi)-\left(I_{l}^{V} \varphi, I_{l-1}^{Q} \psi\right),\left(u_{2}, p_{2}\right)\right) \\
& \quad \leq \operatorname{ch}\left(\|\varphi\|_{2}+\|\psi\|_{1}\right)\left(\left\|u_{2}\right\|_{1}+\left\|p_{2}\right\|_{0}\right) \leq c\left\|u_{2}\right\|_{0} h\left\|I_{l}^{Q} \operatorname{div} u\right\|_{0} .
\end{aligned}
$$

The last term $u_{3}$ is bounded by the triangle inequality.

The discrete divergence free part $u_{1}$ is estimated by lifting to the potential space and Sobolev-Space interpolation in the next lemma.

\section{Lemma 7}

Let $u_{1}$ be defined in (49). Then the estimate

$$
\left\|u_{1}\right\|_{\left[D_{l}, A_{l}\right]_{1 / 2}} \leq c\left\|u_{1}\right\|_{l, 0}
$$

is valid.

Proof: First, we define a lifting procedure $E: V_{l} \rightarrow V$ and a left-inverse interpolation operator $\Pi: V \rightarrow V_{l}$ between discrete divergence free and continuous divergence free functions. Therefore let $X^{+}:=V^{+} \times Q^{+}:=\prod_{T}\left(H_{0}^{1}(T) \times L_{2}(T) / \mathbf{R}\right)$ and set $E u_{1}=u_{1}-w$, with $(w, p) \in X^{+}$such that

$$
B_{0}((w, p),(v, q))=B_{0}\left(\left(u_{1}, 0\right),(0, q)\right) \quad \forall(v, q) \in X^{+} .
$$

Hence, $\operatorname{div} E u_{1}=0$, and by stability and Friedrichs' inequality

$$
\left\|E u_{1}\right\|_{1}+h^{-1}\left\|E u_{1}\right\|_{0} \leq c h^{-1}\left\|u_{1}\right\|_{0} .
$$

Because $\Omega$ is assumed to be convex, there exists a potential $\varphi \in H_{0}^{2}(\Omega)$ such that

$$
E u_{1}=\operatorname{rot} \varphi \quad\|\varphi\|_{2}+h^{-1}\|\varphi\|_{1} \leq c h^{-1}\left\|u_{1}\right\|_{0} .
$$

The interpolation is a modification of the Scott-Zhang interpolation [19]. First, shrink all $\Omega_{i}$ to $\widetilde{\Omega_{i}}=N_{i}+0.9\left(\Omega_{i}-N_{i}\right)$. For each node $N_{i}$ select an edge $e_{i}$ and a set $\sigma_{i}$ such that $N_{i} \in \sigma_{i} \subset e_{i},\left|\sigma_{i}\right| \geq c\left|e_{i}\right|$ and $\sigma_{i} \cap \widetilde{\Omega}_{j}=\emptyset \forall$ corner nodes $N_{i} \neq N_{j}$, see the figure below. We set $\sigma=\cup \sigma_{i}$.

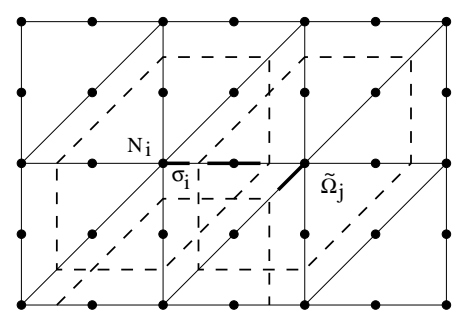

Following [19], we construct a $L_{2}(\sigma)$-biorthogonal basis $\left\{l_{i} \in L_{2}\left(\sigma_{i}\right)\right\}$ to the nodal basis $\left\{p_{i}\right\}$. The projection operator $\Pi_{1}: V \rightarrow V_{l}$

$$
\Pi_{1} v:=\sum\left(v, l_{i}\right)_{L_{2}(\sigma)} p_{i}
$$


is well defined on $H^{1}$ and the approximation is of optimal order

$$
\left\|v-\Pi_{1} v\right\|_{m} \leq c h^{1-m}\|v\|_{1}, \quad m=0,1 .
$$

If $v \in V$ is such that $\operatorname{supp} v \in \tilde{\Omega}_{i}$, then $\Pi_{1} v \in V_{l, i}$. The operator $\Pi_{2}: V \rightarrow V_{l}$ defined by

$$
\begin{aligned}
\Pi_{2} v\left(N_{i}\right)=0 & \forall \text { corner nodes } N_{i} \\
\int_{e_{i}} \Pi_{2} v \mathrm{ds}=\int_{e_{i}} v \mathrm{ds} & \forall \text { edges } e_{i}
\end{aligned}
$$

is standard for Stokes problems [11], p. 211, and fulfills

$$
\left\|\Pi_{2} v\right\|_{1} \leq c\left(\|v\|_{1}+h^{-1}\|v\|_{0}\right) .
$$

Then the projection operator

$$
\Pi_{l}:=\Pi_{2}\left(I-\Pi_{1}\right)+\Pi_{1}
$$

fulfills $\left\|\Pi_{l}\right\|_{1} \leq c$ and $I_{l}^{Q} \operatorname{div} v=I_{l}^{Q} \operatorname{div} \Pi_{l} v$, and thus

$$
\left\|\Pi_{l}\right\|_{A_{l}} \leq c .
$$

Because $E u_{1}-u_{1} \in V^{+}$and $\Pi_{l}$ vanishes on $V^{+}$, it is a left-inverse to the lifting defined above. Because $\Pi_{2}$ preserves support in $\Omega_{i}$, also $\Pi_{l}$ maps $H_{0}^{1}\left(\tilde{\Omega}_{i}\right)$ into $V_{l, i}$.

Let $\left\{\Psi_{i}\right\}$ be a partition of unity fulfilling

$$
\begin{gathered}
\sum \Psi_{i}=1, \quad \operatorname{supp} \Psi_{i} \subset \tilde{\Omega}_{i}, \\
h^{2}\left\|\Psi_{i}\right\|_{2, \infty}+h\left\|\Psi_{i}\right\|_{1, \infty}+\left\|\Psi_{i}\right\|_{0, \infty} \leq c .
\end{gathered}
$$

The product rule and integration by parts gives

$$
\left\|\Psi_{i} \varphi\right\|_{2} \leq c\left(h^{-2}\|\varphi\|_{0, \Omega_{i}}+\|\varphi\|_{2, \Omega_{i}}\right) .
$$

Using $\Pi_{l} \operatorname{rot}\left(\Psi_{i} \varphi\right) \in V_{l, i},(55),(56)$, we get

$$
\begin{aligned}
& \left\|\Pi_{l} \operatorname{rot} \varphi\right\|_{D}^{2} \leq \sum\left\|\Pi_{l} \operatorname{rot}\left(\Psi_{i} \varphi\right)\right\|_{A_{l}}^{2} \leq c \sum\left\|\operatorname{rot}\left(\Psi_{i} \varphi\right)\right\|_{A_{l}}^{2} \leq c \sum\left\|\Psi_{i} \varphi\right\|_{2}^{2} \\
& \quad \leq c \sum\left(h^{-4}\|\varphi\|_{0, \Omega_{i}}^{2}+\|\varphi\|_{2, \Omega_{i}}^{2}\right) \leq c\left(h^{-4}\|\varphi\|_{0}^{2}+\|\varphi\|_{2}^{2}\right)
\end{aligned}
$$

By local $L_{2}$-projection onto a $C^{1}$ continuous FE-space of the same mesh-size $h_{l}$ we can split $\varphi=\varphi_{l}+\tilde{\varphi}$ such that

$$
\left\|\varphi_{l}\right\|_{j} \leq c\|\varphi\|_{j} \quad j=0,1,2
$$

and the inverse inequality

$$
\left\|\varphi_{l}\right\|_{2} \leq c h^{-2}\left\|\varphi_{l}\right\|_{0}
$$

and the approximation inequality

$$
\|\tilde{\varphi}\|_{0} \leq c h^{2}\|\tilde{\varphi}\|_{2}
$$

are fulfilled. This gives $\left\|\Pi_{l} \operatorname{rot} \varphi_{l}\right\|_{D_{l}} \leq c h^{-2}\left\|\varphi_{l}\right\|_{0}$ and $\left\|\Pi_{l} \operatorname{rot} \tilde{\varphi}\right\|_{D_{l}} \leq c\|\tilde{\varphi}\|_{2}$. Using operator interpolation and norm equivalence $H^{1}(\Omega) \sim\left[L_{2}(\Omega), H^{2}(\Omega)\right]_{1 / 2}$ we get

$$
\begin{aligned}
& \left\|u_{1}\right\|_{\left[D_{l}, A_{l}\right]_{1 / 2}}=\left\|\Pi_{l} \operatorname{rot} \varphi\right\|_{\left[D_{l}, A_{l}\right]_{1 / 2}} \leq\left\|\Pi_{l} \operatorname{rot} \varphi_{l}\right\|_{\left[D_{l}, A_{l}\right]_{1 / 2}}+\left\|\Pi_{l} \operatorname{rot} \tilde{\varphi}\right\|_{\left[D_{l}, A_{l}\right]_{1 / 2}} \\
& \quad \leq c\left(\left\|\varphi_{l}\right\|_{\left[h^{-2} L_{2}, H^{2}\right]_{1 / 2}}+\|\tilde{\varphi}\|_{H^{2}}\right) \leq c\left(\left\|\varphi_{l}\right\|_{h^{-1} H^{1}}+\|\tilde{\varphi}\|_{H^{2}}\right) \\
& \quad \leq c\left(h^{-1}\|\varphi\|_{1}+\|\varphi\|_{2}\right) \leq c h^{-1}\left\|u_{1}\right\|_{0} \leq c\left\|u_{1}\right\|_{l, 0} .
\end{aligned}
$$

The component $u_{2}$ is orthogonal to divergence free functions and has non-smooth divergence. 


\section{Lemma 8}

Let $u_{2}$ be defined in (49). Then the estimate

$$
\left\|u_{2}\right\|_{\left[D_{l}, A_{l}\right]_{1 / 2}} \leq c\left\|u_{2}\right\|_{l, 0}
$$

is valid.

Proof: We use $\|\cdot\|_{A_{l}}^{2} \leq c\|\cdot\|_{D_{l}}^{2},\|\cdot\|_{A_{l}}^{2} \leq c h^{-2} \varepsilon^{-1}\|\cdot\|_{0}^{2}$ and the intermediate result (51) to obtain

$$
\begin{aligned}
& \left\|u_{2}\right\|_{\left[D_{l}, A_{l}\right]_{1 / 2}}^{2} \leq c\left\|u_{2}\right\|_{D_{l}}^{2}=c \inf _{u_{2}=\sum u_{i}} \sum\left\|u_{i}\right\|_{A_{l}}^{2} \\
& \leq c \inf _{u_{2}=\sum u_{i}} h^{-2} \varepsilon^{-1}\left\|u_{i}\right\|_{0}^{2} \leq c h^{-2} \varepsilon^{-1}\left\|u_{2}\right\|_{0}^{2} \leq c\|u\|_{l, 0}^{2} .
\end{aligned}
$$

The part $u_{3}$ with smooth divergence will now be estimated by better approximation of the coarse grid interpolant of the dual variable.

\section{Lemma 9}

Let $u_{3}$ be defined in (49). Then the estimate

$$
\left\|u_{3}\right\|_{\left[D_{l}, A_{l}\right]_{1 / 2}} \leq c\left\|u_{3}\right\|_{l, 0}
$$

is valid.

Proof: By definition of $u_{3}$ we have $I_{l}^{Q} \operatorname{div} u_{3}=I_{l-1}^{Q} \operatorname{div} u$, and together with stability of $X_{l-1}$ we get $\left\|u_{3}\right\|_{1} \leq\left\|I_{l-1}^{Q} \operatorname{div} u\right\|_{0}$. This gives

$$
\left\|u_{3}\right\|_{A_{l}}^{2} \leq c\left(\left\|u_{3}\right\|_{1}^{2}+\varepsilon^{-1}\left\|I_{l}^{Q} \operatorname{div} u_{3}\right\|_{0}^{2}\right) \leq c \varepsilon^{-1}\left\|I_{l-1}^{Q} \operatorname{div} u\right\|_{0}^{2} \leq c \varepsilon\left\|u_{3}\right\|_{l, 0}^{2} .
$$

On the other hand, we have

$$
\left\|u_{3}\right\|_{D_{l}}^{2} \leq c \inf _{u_{3}=\sum u_{i}} h^{-2} \varepsilon^{-1}\left\|u_{i}\right\|_{0}^{2} \leq c \varepsilon^{-1} h^{-2}\left\|u_{3}\right\|_{0}^{2} \leq c \varepsilon^{-1}\left\|u_{3}\right\|_{l, 0}^{2} .
$$

By operator interpolation we finish the proof.

\section{$7 \quad$ Numerical Results}

Several versions of the multigrid method in primal variables developed and analyzed above have been tested numerically. The following two problems have been investigated within the finite element code FEPP on a SUN Ultra 1 / $166 \mathrm{MHz}$ workstation with $320 \mathrm{MB}$ RAM.

\section{Problem A: Driven Cavity example.}

We consider the unit square $\Omega=(0,1)^{2}$. The initial triangulation $\mathcal{T}_{1}$ is given by two triangles, further meshes are obtained by successive refinement. We have used the finite element space based on $P_{2}$ elements. The bilinear-form $A_{L}(.,$.$) on the finest level is defined$ in (8), where the projection $I_{L}^{Q}$ maps into the piece-wise constant FE-space. The source term is set to $f=0$. Dirichlet boundary conditions are specified as

$$
u_{L}= \begin{cases}(1,0)^{T} & \text { at nodes } \in[0,1] \times\{1\}, \\ (0,0)^{T} & \text { at nodes } \notin[0,1] \times\{1\},\end{cases}
$$




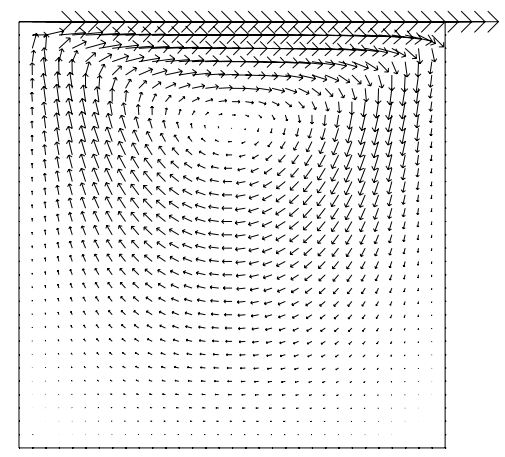

Figure 4: Solution of Problem A

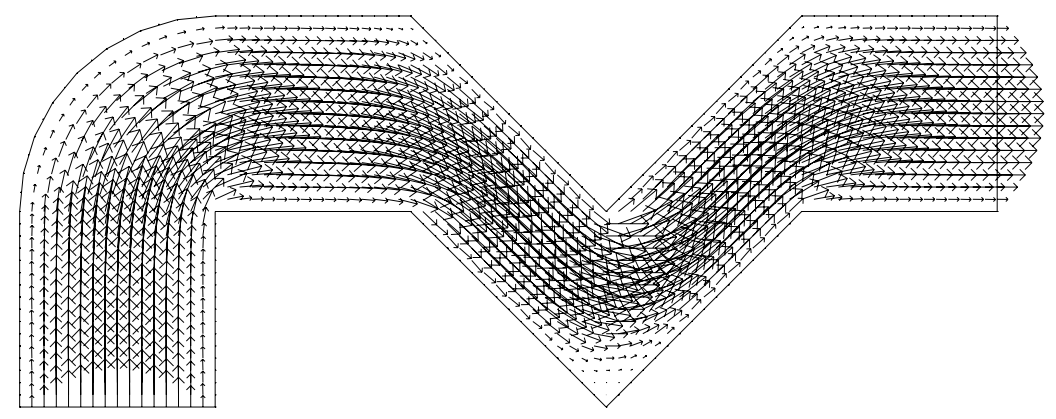

Figure 5: Solution of Problem B

and incorporated by homogenization of the FE system. A plot of the solution at level 5 is given in Figure 4.

\section{Problem B: Flow through a pipe.}

The geometry and the solution at level 4 are given in Figure 5. The boundary is split into the jacket $\Gamma_{1}$, inlet boundary $\Gamma_{2}$ and outlet boundary $\Gamma_{3}$. We specify homogeneous Dirichlet boundary conditions at $\Gamma_{1}$ and natural boundary conditions elsewhere. We solve the finite element problem find $u_{L} \in V_{L}$ such that

$$
\tilde{A}_{L}\left(u_{L}, v_{L}\right)=\left(g, v_{L}\right)_{0, \Gamma_{2}} \quad \forall v_{L} \in V_{L} .
$$

The bilinear-form $\tilde{A}(u, v)$ is obtained from $A(u, v)$ by replacing the term $(e(u), e(v))_{0}$ by $(\nabla u, \nabla v)_{0}$. This is done to obtain physically correct boundary conditions. The boundary stress is defined as $g=(0,1)^{T}$. The problem involves curved boundary approximation, a non-convex domain and mixed boundary conditions.

At first, we investigate the behavior of the condition number $\kappa\left(C_{L}^{-1} A_{L}\right)$ in dependence of the number of levels $L$ and the parameter $\varepsilon$. The preconditioner $C_{L}$ is obtained by the application of a symmetric multigrid operator, either a W-2-2 cycle or a V-1-1 cycle. In addition to the additive smoother (16), we use the multiplicative counterpart

$$
S_{l}^{A}=\prod_{i=1}^{n_{l}}\left(I-P_{l, i}^{A_{l}}\right)
$$

for pre-smoothing and in reversed order for post-smoothing. It does not need damping at all. The numerical results for the condition number $\kappa\left(C_{L}^{-1} A_{L}\right)$ for Problem A obtained by 


\begin{tabular}{|c|c|l|l|l|l|l|l|l|l|}
\hline$l$ & Unknowns & \multicolumn{4}{|c|}{ additive smoother } & \multicolumn{3}{|c|}{ multiplicative smoother } \\
\hline \multicolumn{2}{|r|}{$\varepsilon=$} & $10^{0}$ & $10^{-2}$ & $10^{-4}$ & $10^{-6}$ & $10^{0}$ & $10^{-2}$ & $10^{-4}$ & $10^{-6}$ \\
\hline 2 & 50 & 1.82 & 2.51 & 2.66 & 2.66 & 1.04 & 1.10 & 1.11 & 1.11 \\
3 & 162 & 2.27 & 6.79 & 7.66 & 7.67 & 1.26 & 2.15 & 2.29 & 2.30 \\
4 & 578 & 2.58 & 8.59 & 9.91 & 9.93 & 1.37 & 2.47 & 2.64 & 2.64 \\
5 & 2178 & 2.72 & 9.79 & 11.60 & 11.62 & 1.39 & 2.56 & 2.73 & 2.73 \\
6 & 8450 & 2.79 & 10.84 & 13.12 & 13.15 & 1.39 & 2.65 & 2.82 & 2.82 \\
7 & 33282 & 2.73 & 11.66 & 14.41 & 14.45 & 1.39 & 2.72 & 2.90 & 2.91 \\
\hline
\end{tabular}

Table 1: Condition numbers for V-1-1 cycle

\begin{tabular}{|c|c|l|l|l|l|l|l|l|l|}
\hline$l$ & Unknowns & \multicolumn{3}{|c|}{ additive smoother } & \multicolumn{4}{|c|}{ multiplicative smoother } \\
\hline \multicolumn{2}{|r|}{$\varepsilon=$} & $10^{0}$ & $10^{-2}$ & $10^{-4}$ & $10^{-6}$ & $10^{0}$ & $10^{-2}$ & $10^{-4}$ & $10^{-6}$ \\
\hline 2 & 50 & 1.05 & 1.08 & 1.10 & 1.10 & 1.000 & 1.00 & 1.00 & 1.00 \\
3 & 162 & 1.15 & 1.65 & 1.74 & 1.74 & 1.002 & 1.05 & 1.06 & 1.06 \\
4 & 578 & 1.19 & 1.76 & 1.73 & 1.73 & 1.002 & 1.05 & 1.05 & 1.06 \\
5 & 2178 & 1.24 & 1.79 & 1.87 & 1.86 & 1.002 & 1.04 & 1.05 & 1.05 \\
6 & 8450 & 1.26 & 1.86 & 1.92 & 1.91 & 1.002 & 1.05 & 1.05 & 1.05 \\
7 & 33282 & 1.26 & 1.87 & 1.92 & 1.92 & 1.002 & 1.05 & 1.05 & 1.05 \\
\hline
\end{tabular}

Table 2: Condition numbers for W-2-2 cycle

the Lanzcos method are given in Table 1 for a V-1-1 cycle and in Table 2 for a W-2-2 cycle. For the W-2-2 the calculated condition numbers neither depend on the level nor on the parameter, what is in correspondence with the analysis provided. We do not have optimal estimates for $\mathrm{V}$-cycle convergence rate yet, but the numerical results seem to be very promising.

Next, we used the V-1-1 multigrid preconditioner in a preconditioned conjugate gradients solver for the solution of Problem A and Problem B. The small parameter is set to $\varepsilon=10^{-6}$. The iteration is terminated after an reduction of the error in energy norm by a factor of $10^{8}$. The necessary iteration numbers and CPU times are shown in Table 3 and Table 4, respectively.

\begin{tabular}{|c|c|c|c|}
\hline Level & Unknowns & Iterations & Time[sec] \\
\hline 2 & 50 & 4 & 0.01 \\
3 & 162 & 10 & 0.08 \\
4 & 578 & 15 & 0.41 \\
5 & 2178 & 15 & 1.88 \\
6 & 8450 & 16 & 8.56 \\
7 & 33282 & 16 & 37.06 \\
8 & 132098 & 16 & 154.80 \\
\hline
\end{tabular}

Table 3: Iteration numbers and CPU times for Problem A, PCG with V-1-1 cycle 


\begin{tabular}{|c|c|c|c|}
\hline Level & Unknowns & Iterations & Time[sec] \\
\hline 2 & 230 & 10 & 0.1 \\
3 & 810 & 13 & 0.6 \\
4 & 3026 & 15 & 2.7 \\
5 & 11682 & 17 & 12.9 \\
6 & 45890 & 18 & 58.2 \\
7 & 181890 & 18 & 242.0 \\
\hline
\end{tabular}

Table 4: Iteration numbers and CPU times for Problem B, PCG with V-1-1 cycle

Finally, I want to express my thanks to D. Braess, U. Langer and Ch. Wieners for many discussions on this topic and for valuable suggestions to improve the presentation.

\section{References}

[1] D. Braess. Stability of saddle point problems with penalty. $M^{2} A N, 30: 731-742,1996$.

[2] D. Braess. Finite Elements: Theory, Fast Solvers, and Applications in Solid Mechanics. Springer, Berlin, Heidelberg, New York, 1997.

[3] D. Braess and C. Blömer. A multigrid method for a parameter dependent problem in solid mechanics. Numer. Math., 57:747-761, 1990.

[4] D. Braess and R. Sarazin. An efficient smoother for the Stokes problem. Appl. Num. Math., 23:3-19, 1997.

[5] J. H. Bramble. Multigrid Methods. Longman Scientific \& Technical, Longman House, Essex, England, 1993.

[6] J. H. Bramble, J. E. Pasciak, and J. Xu. The analysis of multigrid algorithms with non-imbedded or non-inherited quadratic forms. Math. Comp., 55:1-34, 1991.

[7] S. Brenner and L. R. Scott. The Mathematical Theory of Finite Element Methods. Springer, Berlin, Heidelberg, New York, 1994.

[8] S. C. Brenner. A nonconforming multigrid method for the stationary Stokes equations. Mathematics of Computations, 55(192):411-437, 1990.

[9] S. C. Brenner. Multigrid methods for parameter dependent problems. Math. Mod. a. Num. Anal., 30:265-297, 1996.

[10] S. C. Brenner and L.-Y. Sung. Linear finite element methods for planar linear elasticity. Math. Comp., 59(200):321-338, 1992.

[11] F. Brezzi and M. Fortin. Mixed and Hybrid Finite Element Methods. Springer, Berlin, Heidelberg, New York, 1991.

[12] R. S. Falk. Nonconforming finite element methods for the equations of linear elasticity. Mathematics of Computation, 57:529-550, 1991.

[13] V. Girault and P. Raviart. Finite Element Methods for Navier-Stokes Equations. Springer, Berlin, Heidelberg, New York, 1986. 
[14] W. Hackbusch. Multi-Grid Methods and Applications. Springer, Berlin, Heidelberg, New York, 1985.

[15] Z. Huang. A multi-grid algorithm for mixed problems with penalty. Numer. Math., $57: 227-247,1990$.

[16] J. Lions and J. Peetre. Sur une classe d'espaces d'interpolation. Institut des Hautes Etudes Scientifique, Publ.Math., 19:5-68, 1964.

[17] P. Oswald. Multilevel Finite Element Approximation. Teubner Stuttart, 1994.

[18] J. Schöberl. Multigrid Methods for Parameter Dependent Problems. In W. Hackbusch and G. Wittum, editors, Proceedings of the Fifth European Multigrid Conference 1996. Springer, 1998. (to appear)

[19] L. R. Scott and S. Zhang. Finite element interpolation of nonsmooth functions satisfying boundary conditions. Math. Comp., 54(190):483-493, 1990.

[20] S. Turek. Multigrid techniques for a divergence-free finite element discretization. EastWest J.Numer.Math., 2(3):229-255, 1994.

[21] R. Verfürth. A multilevel algorithm for mixed problems. SIAM J. Numer. Anal., 21:264-271, 1984.

[22] J. Xu. Iterative Methods by Space Decomposition and Subspace Correction. SIAM Review, 34(4):581-613, 1992. 